\title{
Hippocampus modulates self-stimulation reward from the ventral tegmental area in the rat
}

\author{
LEO VAN WOLFSWINKEL and JAN M. VAN REE \\ Rudolf Magnus Institute for Pharmacology, Medical Faculty, University of Utrecht, 3521 GD Utrecht (The Netherlands)
}

(Accepted June 26th, 1984)

Key words: hippocampus - self-stimulation — ventral tegmental areat

\begin{abstract}
Rats with electrodes in the ventral tegmental area were tested for the threshold of intracranial reward using a rate insensitive selfstimulation procedure. It was found that an electrolytic lesion of a part of the dorsal hippocampus induced a marked decrease in the variation of thresholds across rats, while the mean reward level did not change. This indicates that a factor is removed, by the hippocampal lesion, which causes differences in reward between individual rats. It is suggested that the mesolimbic dopaminergic system is involved in this modulatory influence of the hippocampus on reward.
\end{abstract}

It has been suggested that mechanisms present in the hippocampus interact with brain reward systems ${ }^{1,2}$. An exaggerated reaction to signalled administration of food reward has been described in rats with extensive bilateral hippocampal damage ${ }^{3}$. This reaction, which includes behavioral stereotypy and increased locomotor activity, bears resemblance with amphetamine-induced behavioral changes and is blocked by the dopamine antagonist haloperidol. These observations were interpreted as indicating that the intact hippocampus moderates the effect of reward, probably by opposing catecholamine mechanisms ${ }^{3}$. Indeed, hippocampal damage has been found to influence dopaminergic ascending systems as assessed by behavioral and biochemical methods ${ }^{8.10}$. These systems include the mesolimbic dopaminergic system with cell bodies in the ventral tegmental area and terminals in a number of limbic structures, e.g. the nucleus accumbens ${ }^{11}$. This system is thought to be important for reward, especially as assessed with electrical intracranial self-stimulation (ICSS) ${ }^{13}$. However, the interaction between the hippocampus and the dopaminergic systems is not well understood, since hippocampal damage has been reported to result in both increased and decreased dopaminergic activity ${ }^{10.14 .15}$. Moreover, experimental evidence for a direct interaction between the hippocampus and brain reward is scarce. The present experiment was designed to investigate whether damage of the hippocampus directly affects brain reward elicited in the mesolimbic system and assessed with ICSS. The performance of well trained rats with an electrode in the ventral tegmental area was compared before and after electrolytic hippocampal lesions using a response rate insensitive self-stimulation schedule 9.12 .

Ten male Wistar rats (TNO, Zeist, The Netherlands) from our own breeding stock weighing approximately $230 \mathrm{~g}$ at the time of the operation were kept in single transparent cages under standard conditions with laboratory food and tap water available ad libitum throughout the experiment. Under Hypnorm anesthesia $(0.7 \mathrm{ml} / \mathrm{kg}$ of the solution containing $0.2 \mathrm{mg} / \mathrm{ml}$ fentanyl and $10 \mathrm{mg} / \mathrm{ml}$ fluanison) they were implanted with a bipolar stainless steel stimulation electrode of $200 \mu \mathrm{m}$ thickness that was insulated except at the cross section at the tip, in the ventral tegmental area (coordinates: A $2.6 ; \mathrm{D}-3.7$ : $\mathrm{L} 1.0$ according to De Groot, (see ref. 7) and a monopolar lesion electrode of $200 \mu \mathrm{m}$ thickness in the ipsilateral dorsal hippocampus (coordinates: A 2.6; D $0.3 ; \mathrm{L}$ $3.0)$. One $\mathrm{mm}$ at the tip of this electrode was not insulated. The electrodes were fixed to screws in the skull

Correspondence: J. M. van Ree, Rudolf Magnus Institute for Pharmacology, Medical Faculty. University of Utrecht, Vondellaan 6. 3521 GD Utrecht. The Netherlands. 
with acrylic dental cement and the rats were allowed to recover from the operation for at least 1 week.

The experiment was performed in a standard Skinner box, made from aluminium with the size $23 \times 25$ $\times 20 \mathrm{~cm}$ (length $\times$ width $\times$ height) and with a plexiglass front door (Campden Instruments Ltd, U.K.). Two side by side levers were present, $6 \mathrm{~cm}$ above the stainless steel grid floor and $14.5 \mathrm{~cm}$ apart. A spring shielded lead and a mercury swivel connected the electrode to the stimulator (type ST, Janssen Scientific Instruments, Belgium). The stimulator produced $500 \mathrm{~ms}$ trains of biphasic rectangular pulses with a frequency of $100 \mathrm{~Hz}$ and a pulse duration and interval between the positive and negative pulse of $0.5 \mathrm{~ms}$. The experimental setup was controlled by standard $24 \mathrm{~V}$ electromechanical equipment and a PDP 8 computer using SCAT software. The rats were trained to perform a behavioral self-stimulation procedure in which a response contingent stimulus to the stimulation electrode, which decreased $1 \%$ of the maximal current after every response, could be reset to the maximal current any time by a response on the second lever. In 7 rats no stimulation was available during $5 \mathrm{~s}$ after every reset response, the other 3 rats were tested without this time-out. The maximal current was individually adjusted to the performance of the rats and ranged from 150 to $220 \mu \mathrm{A}$. The current intensity at which the rat reset the current was recorded and the mean reset current of the $25 \mathrm{~min}$ test session was considered as the threshold current. This threshold current was expressed as a percentage of the maximal current (relative threshold). The rats were trained until their performance was stable. Then, baseline performance was recorded during at least 3 days. Thereafter, in 4 rats a radiofrequency electrolytic lesion was made via the hippocampal electrode (12 mA, $120 \mathrm{~s})$ with a Grass lesion maker under ether anaesthesia. Six rats served as controls and were treated identically but no current was applied to the hippocampal electrode (sham lesions). From the day after the lesion or the sham lesion the self-stimulation threshold was recorded again for at least 3 days. When stable, the control rats were also lesioned and their performance was determined for at least 3 days.

After completion of the experiment the rats were decapitated, their brains quickly removed and stored in $4 \%$ formalin. The location of the electrode and the site and extension of the lesion were verified in frozen sections of $100 \mu \mathrm{m}$ thickness, stained with thionin.

To analyze the effect of the lesion the mean relative threshold and the response rate of the 3 sessions before the (sham) lesion were compared to the mean threshold and response rate on the 3 days after the (sham) lesion. Statistical analysis involved analysis of variance with repeated measures and an $F$-test for homogeneity of variance.

The tips of the stimulation electrodes appeared to be in the ventral tegmental area on the border of the substantia nigra, pars compacta (Fig. 1). Histological analysis of the lesion site revealed that in the medial part of the dorsal hippocampus at the level of the hippocampal curvature a spherical lesion was formed with a diameter of $2-3.5 \mathrm{~mm}$. This lesion involved the dorsal hippocampus (dentate gyrus and part of the CA3 and CA1 fields). The cortex and other surrounding structures were hardly damaged (Fig. 1). The response rate per minute was calculated from the number of rewarded responses and the time during which stimulation was available to the rat in a session. The mean response rate ( \pm S.E.M.) was $44 \pm 4$ responses/min. The number of reset responses per 25 -min session ranged from 20 to 50 when the reset response was followed by a time-out, and was about 120 when no time-out was available.

Typical examples of the effect of hippocampal lesions on the relative threshold for self-stimulation behavior are shown in Fig. 2. Sham lesions did not affect the rate of self-stimulation or the threshold at which the current was reset. Actual lesions increased or decreased the threshold. This change was in some rats constant for as long as the threshold was measured (up to 3 weeks) (rat A, Fig. 2), while in other animals the threshold returned to the pre-lesion level within 4-7 days (rat B, Fig. 2). The effect of the lesion in rats that had been sham lesioned before they were actually lesioned was not different from the effect in rats that had been lesioned without the preceding sham lesion procedure. The data of all lesioned rats have therefore been combined. In rats that were both lesioned and sham lesioned the procedures were considered as statistically independent cases. The absence of the time-out procedure caused a significantly higher threshold $\left(F_{1.12}=14.7, P<0.01\right)$ but no differences in response rate were present in rats with 

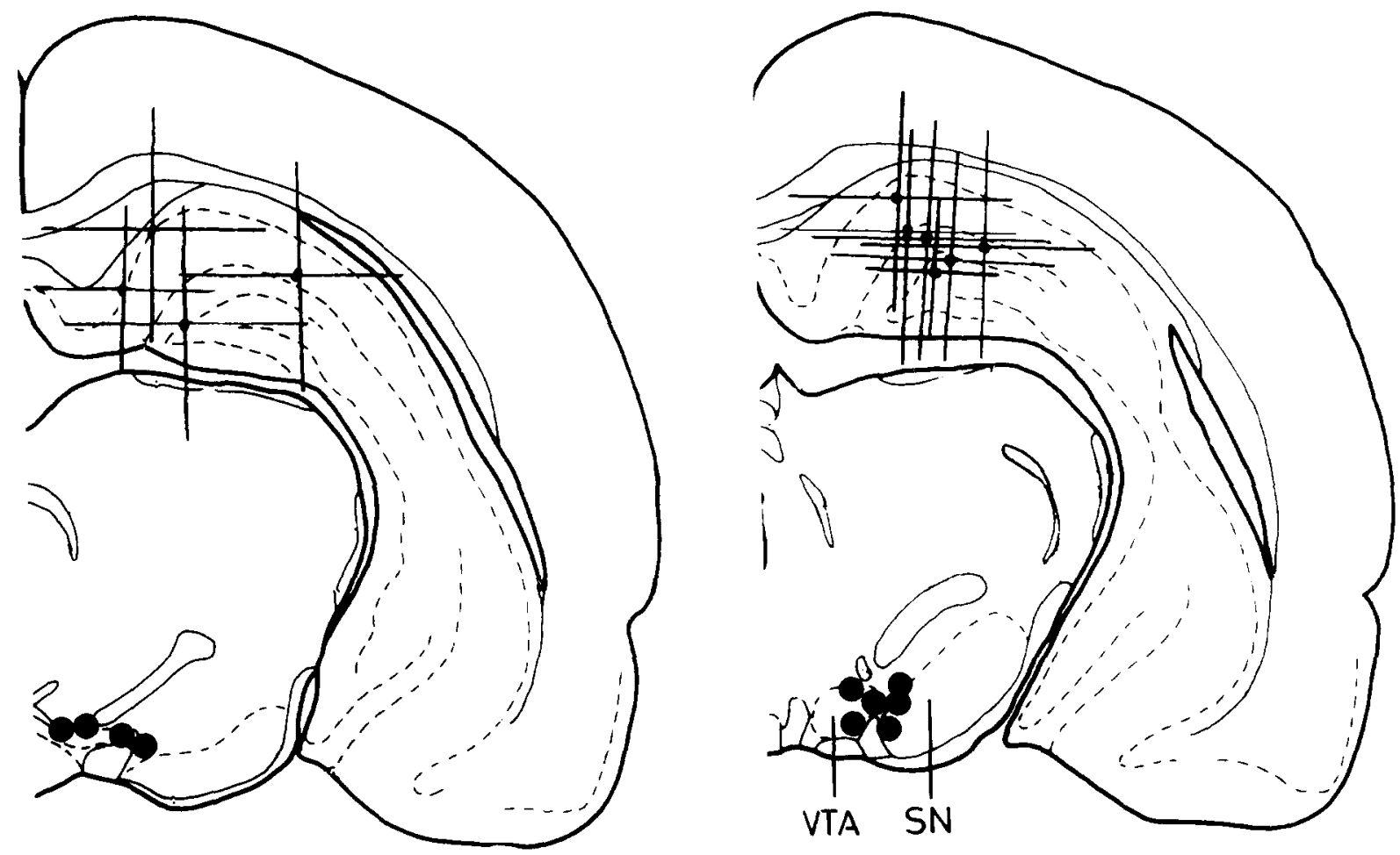

Fig. 1. Histological verification of placement of stimulation electrodes in the ventral tegmental area and of the location and the size of electrolytic lesions produced by the hippocampal electrode. Sections have been redrawn from Pellegrino ${ }^{7} \bullet$. Location of tip of stimulation electrode. +, Midpoint of hippocampal lesions. The extension of the lesion is indicated by the crossing lines. VTA, ventral tegmental area; $\mathrm{SN}$, substantia nigra.

and without the time-out procedure. No interaction of time-out with lesion-treatment was found on both threshold and response rate. Thus, the thresholds of rats without time-out were corrected for the mean difference between the relative thresholds of the

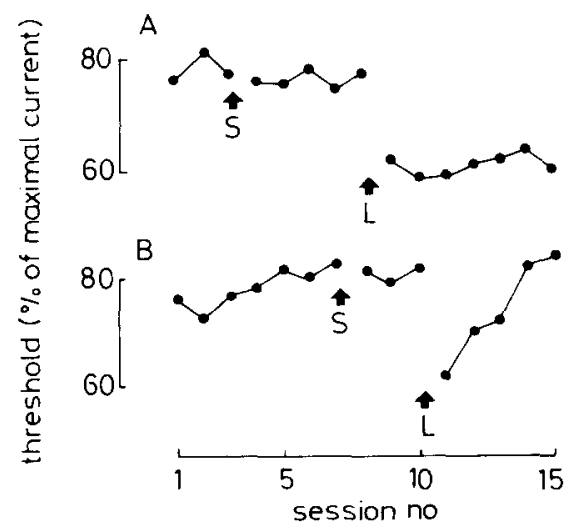

Fig. 2. Reward thresholds of rats before and after a sham lesion (S) and an actual lesion (L) in the hippocampus. Two typical examples are shown: the decreased threshold did not return to the pre-lesion level (A); the threshold returned to the pre-lesion levels in 4 sessions (B). time-out and no time-out rats, and they were considered as one group, with adjustment of degrees of freedom. The mean relative threshold of both the control and the lesion group was not significantly different before and after the lesion procedure (difference, $F_{1,12}=0.6$; interaction with lesion procedure, $F_{1,12}=0.5, P>0.2$ ). However, as depicted in Fig. 3 , the change in threshold in individual rats was large in the lesioned animals while the control rats had a constant threshold ( $F$-test comparing variances of differences between pre- and post-treatment of lesioned and sham-lesioned rats: $F(\max )_{2,5}=26.11$, $P<0.001$ ). It was observed that the 5 rats with the highest relative thresholds had decreased thresholds after the lesion, with one exception, while the 5 lowest thresholds were increased, resulting in a convergence of all thresholds into a narrow region around $68 \%$ of the maximal current. Thus, the variance of the relative thresholds after the lesion is much less than that before the lesion $\left(F(\max )_{2,8}=5.96\right.$, $P<0.05$, sham lesioned rats: $F(\max )_{2,4}=1.15$, $P>0.2$. This finding of the narrowed threshold 


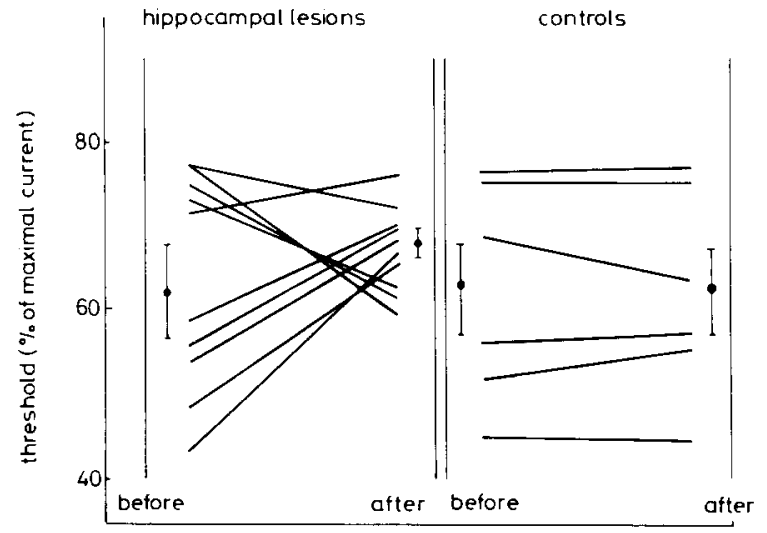

Fig. 3. Effect of ipsilateral hippocampal lesions on a response rate independent reward threshold of individual rats. The mean of the 3 sessions after the lesion are compared to the 3 days before the lesion. Rats with high thresholds had a lower threshold after the actual lesion, while those with a low threshold had a higher threshold after the lesion. The mean threshold did not change but the variance decreased significantly $(P<0.05)$. Sham lesions were not followed by a change in threshold. Also shown are the mean thresholds of the groups before and after the procedures. Vertical bars indicate S.E.M.

range was further analyzed in the consecutive 5-min blocks of each 25-min session (Fig. 4). The pattern of the mean reset level over time was the same before and after the lesion: in the first $5 \mathrm{~min}$ the threshold was lower than thereafter ( 5 min periods within rats: $\left.F_{4,9}=9.27, P<0.005\right)$. This threshold change over time was not influenced by the lesion $\left(F_{4,9}=2.30\right.$, $P>0.1)$. The variance of the threshold between rats

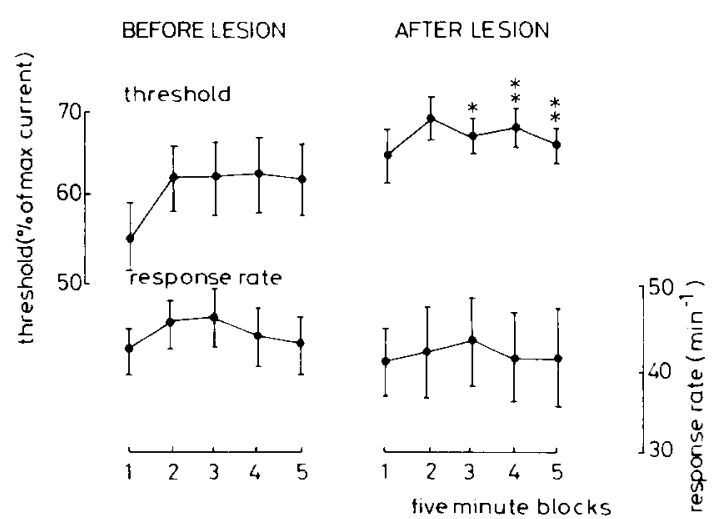

Fig. 4. Analysis of thresholds and response rates in the 5-min periods of the 25 -min self-stimulation session (shown are the means of 3 sessions). The variance of the threshold between rats decreased after the actual lesion, while the variance in response rate was not changed by the lesion procedure. ${ }^{*} P<0.05,{ }^{* *} P<0.01, F$-test comparing the variance between the corresponding 5 -min periods before and after the actual hippocampal lesion. before the lesion was constant over time, while after the treatment a decrease of this variance was observed.

The mean response rate and the variance in this rate did not change after the lesion or the sham lesion, but the individual changes in response rate, either an increment or a decrement were large in the lesioned rats as compared to the sham lesioned rats $\left(F(\max )_{2,5}=40.5, P<0.01\right)$. Although in individual rats both the response rate and the threshold changed after the lesion, no correlation was found between these changes $(r=-0.51, \mathrm{n}=10, P>0.10)$.

The present findings indicate that the hippocampus is involved in reward elicited by electrical stimulation of the mesolimbic system. The reward is modulated by the hippocampus, as a lesion of this structure decreased the reward threshold in rats with a high threshold, while this threshold was increased in the other rats. Thus, the variance of the threshold between lesioned rats was small compared to the variance before the lesion. It appears that this effect on reward can be achieved by a relatively small lesion of the dorsal hippocampus. Extensive damage is not necessary, suggesting that only a part of the hippocampus may be involved in this regulation. It has been argued before that threshold determination is relatively insensitive to changes in motor performance of the rats and that this threshold is a more accurate variable to measure reward than response rate $^{9,12}$. This is stressed by the fact that, although in lesioned rats the changes in both the response rate and the threshold are much greater than in sham lesioned rats, these changes are not correlated. Also, the response rate decrease found in the last part of the session, which decrease is probably due to fatigue, is not accompanied by a change in threshold, while in the first period of the session the lower threshold is attended with a low response rate. Thus, a change in threshold in the present procedure is mainly related to changes in reward and less, if any, to changes in motor performance.

The lesion electrodes were placed on the same side of the brain as the stimulation electrode. Although extensive interconnections exist between both sides of the hippocampus, parts of the mesolimbic system that are important for reward, like the nucleus accumbens, are mainly innervated by ipsilateral projections $^{6}$. Thus, the fibers to the nucleus accumbens, 
that pass through the fornix, part of which was also damaged in the present experiment, are probably involved in the influence of the hippocampus on reward. It has previously been reported that hippocampal lesions can alter reward-related locomotion and stereotypy in rats and change the dopamine metabolism in the nucleus accumbens and the striatum ${ }^{3,10}$. Since dopamine is probably involved in reward as the most important neurotransmitter ${ }^{5}$, the connection between the hippocampus and the dopaminergic mesolimbic system may mediate the modulatory activity of the hippocampus on reward. However, it cannot be excluded that this influence is on other structures involved in the general mechanism of processing of information and the perception (recognition) of reward. Nevertheless, somehow the intact hippocampus causes variation in reward measured with the rate independent threshold. The variability in reward between rats before the lesion may partly be caused by minor individual differences in the location and the shape of the electrodes and in stimulation parameters, but also by different experiences of individual

1 Deadwyler, S. A., West, M. and Lynch, G., Activity of dentate granule cells during learning: differentiation of perforant path input, Brain Research, 169 (1979) 29-43.

2 Deadwyler, S. A., West, M. O. and Robinson, J. H., Entorhinal and septal inputs differentially control sensoryevoked responses in the dentate gyrus, Science, 211 (1981) 1181-1183.

3 Devenport, L. D., Devenport, J. A. and Holloway, F. A., Reward-induced stereotypy: modulation by the hippocampus, Science, 212 (1981) 1288-1289.

4 Devenport, L. D. and Holloway, F. A., The rats resistance to superstition: role of the hippocampus, J. comp. Physiol. Psychol., 94 (1980) 691-705.

5 German, D. C, and Bowden, D. M., Catecholamine systems as the neuronal substrate for intracranial self-stimulation: a hypothesis, Brain Research, 73 (1974) 381-419.

6 Isaacson, R. L., The Limbic System, 2nd edn., Plenum Press, New York, 1982, 327 pp.

7 Pellegrino, L. J., Pellegrino, A. S. and Cushman, A. J., A Stereotaxic Atlas of the Rat Brain, 2nd edn., Plenum Press, New York, 1979, $280 \mathrm{pp}$.

8 Reinstein, D. K., Hannigan, J. H. and Isaacson, R. A., Time course of certain behavioral changes after hippocampal damage and their alteration by dopaminergic intervention into the nucleus accumbens, Pharmacol. Biochem. Behav., 17 (1982) 193-202 rats during training and further testing. One function of the hippocampus is the processing of incoming information in order to optimize behavior. It thus induces behavioral variability in the sense that animals more readily change their behavioral strategies depending on the situation ${ }^{4}$. Reduction of this modulatory influence by a hippocampal lesion may reduce the effect of information-containing stimuli and may lead to less variation in behavior between rats. In this view, the involvement of the hippocampus in reward processes is that differences in rewarding value of the stimulus are at least partly mediated through this structure. These differences are caused by experiences that are not strictly dependent on the rewarding stimulus. Some of these experiences apparently increase the rewarding value of the stimulation while other stimuli decrease this reward. The hippocampus may thus function as a structure that modulates behavior to facilitate adaptation to changes in environmental circumstances. The present study suggests that this modulation can be extended to brain reward.

9 Schaefer, G. J. and Holtzman, S. G., Free operant and auto-titration brain self-stimulation procedures in the rat: a comparison of drug effects, Pharmacol. Biochem. Behav., $10(1979) 127-135$.

10 Springer, J. E. and Isaacson, R. L., Catecholamine alterations in basal ganglia after hippocampal lesions, Brain Research, 252 (1982) 185-188.

11 Swanson, L. W. and Cowan, W. M., An autoradiographic study of the organization of the efferent connections of the hippocampal formation in the rat, $J$. comp. Neurol., 172 (1977) 49-84

12 Van Wolfswinkel, L. and Van Ree, J. M., Effects of morphine and naloxone on ventral tegmental electrical selfstimulation. In F. C. Colpaert and J. F. Slangen (Eds.), Drug Discrimination: Applications in CNS Pharmacology, Elsevier, Amsterdam, 1982, pp. 391-397

13 Wise, R. A., Brain neuronal systems mediating reward processes. In J. E. Smith and J. D. Lane (Eds.), The Neurobiology of Opiate Reward Processes, Elsevier, Amsterdam, 1983 , pp. $405-438$.

14 Woodruff, M. L., Attenuation of the rate-decreasing effects of amphetamine on fixed-ratio responding in rats by hippocampal lesions, Behav. Biol., 20 (1977) 493-499.

15 Woodruff, M. L., Baisden, R. H. and Fish, B. S., Forebrain catecholamine concentrations in the rat after neocortical and hippocampal lesions, Life Sci., 29 (1981) 2603-2607. 